\title{
Liberalização da conta de capitais e crescimento econômico: evidências de dados em painel para a América Latina
}

ADERBAL OLIVEIRA DAMASCENO*

Capital account liberalization and economic growth: evidences from data in Latin-American panel. The aim of this paper is to carry out an empirical analysis about the relation between Capital Account Liberalization and Economic Growth having as object of study the experience of 16 countries of Latin America with annual data for the period 1986-2000. The econometrical calculations do not corroborate the hypothesis that the liberalization of Capital Account would stimulate the economic growth. The results suggest an adverse effect of the liberalization of the Capital Account on the real growth gross domestic product per capita of the countries.

Keywords: capital account liberalization; economic growth; Latin America.

JEL Classification: F33; F36; F43.

\section{INTRODUÇÃO}

O objetivo deste trabalho é realizar uma investigação empírica acerca da relação entre Liberalização da Conta de Capitais e Crescimento Econômico tendo como objeto de estudo a experiência da América Latina no período 1986-2000. Como metodologia econométrica utiliza-se um modelo dinâmico de dados em painel, estimado, fazendo-se uso da abordagem proposta originalmente por Arellano e Bond (1991).

O estudo da experiência da América Latina tem como motivação, principalmente, o fato de que os países da região, desde o final da década de 1980 e mais acentuadamente ao longo da década de 1990, moveram-se em direção a uma

*Aderbal Oliveira Damasceno é professor do Instituto de Economia da Universidade Federal de Uberlândia (IE-UFU). E-mail: damasceno@ie.ufu.br. Submetido: Setembro 2006; Aprovado: Março 2007. 
maior liberalização da conta de capitais, bem como, após uma década de escassez, receberam grande fluxo de capitais externos.

O trabalho está organizado em quatro seções além desta introdução; i) na seção 2 é feita uma revisão da literatura teórica e empírica acerca da relação entre Liberalização da Conta de Capitais e Crescimento Econômico; ii) na seção 3 é especificado o modelo a ser estimado e apresentada a abordagem econométrica utilizada; iii) na seção 4 são apresentados e discutidos os resultados dos cálculos econométricos; iv) e, por fim, na seção 5 , alinham-se algumas considerações finais.

\section{LIBERALIZAÇÃO DA CONTA DE CAPITAIS E CRESCIMENTO ECONÔMICO}

\section{Debate teórico}

No âmbito da literatura convencional, modelos teóricos sublinham um conjunto de canais através dos quais a liberalização da conta de capitais levaria à melhoria na eficiência da alocação de recursos, estimulando o crescimento econômico dos países. No que segue, esses canais são apresentados resumidamente.

Alocação Internacional de Recursos - A livre mobilidade de capitais permitiria que o capital fluísse de países onde é relativamente abundante e a produtividade marginal do capital é baixa (economias desenvolvidas) para países onde o capital é relativamente escasso e a produtividade marginal do capital é alta (economias em desenvolvimento), aumentando a eficiência na alocação internacional de recursos. Decorre daí que a liberalização da conta de capitais seria particularmente benéfica para os países em desenvolvimento, tendo como resultado o aumento da poupança doméstica, aumento da acumulação de capital e maior crescimento econômico. ${ }^{1}$

Melhor Alocação Global de Riscos - A mobilidade internacional de capitais criaria a oportunidade para a diversificação de portfolio e compartilhamento de riscos, o que permitiria aos investidores obterem altas taxas de retorno ajustadas ao risco, estimulando decisões de investimento e resultando em maiores taxas de crescimento econômico. Ou seja, como argumentam Eichengreen et al. (1998), "Capital mobility can thereby enable investors to achieve higher risk-adjusted rates of return. Income levels in recipient countries should also rise as a result of the capital inflows. Higher rates of return can encourage saving and investment that deliver faster rates of economic growth".

Transferência de Tecnologia — Prasad et al. (2003: 25) argumentam que eco-

\footnotetext{
${ }^{1}$ Obstfeld e Taylor (2004: 9) deixam claro este argumento: “A country that has rich investment opportunities, but that generates little saving of its own, can tap the international capital market to exploit its investment potential without massive short-run consumption cutbacks. Conversely, countries with abundant savings but more limited investment prospects at home can earn higher returns to wealth than they would domestically. Both borrowers and lenders gain as capital flows to its most productive uses worldwide. In particular, developing countries can invest more than they could if closed [...] The process of economic convergence is hastened by capital flows from rich to poor countries".
} 
nomias com contas de capitais liberalizadas tendem a atrair grande parte dos fluxos de capitais na forma de investimento externo direto, "which have the potential to generate technology spillovers and to serve as a conduit for passing on better management practices. These spillovers can raise aggregate productivity and, in turn, boost economic growth".

Desenvolvimento do Sistema Financeiro Doméstico - A liberalização da conta de capitais incrementaria a eficiência do sistema financeiro doméstico devido à possibilidade de especialização na oferta de serviços financeiros e à maior concorrência entre instituições residentes e não-residentes. Em adição, a integração com o mercado financeiro internacional reduziria a margem de intermediação e abriria acesso ao mercado externo, possibilitando a obtenção de créditos com menores custos por empresas dos países em desenvolvimento (Agénor, 2003; Einchengreen et al. 1998, Klein \& Olivei, 1999: 1).

Disciplina Macroeconômica - Tem sido argumentado que, por aumentar a recompensa para a prática de boas políticas econômicas (disciplina fiscal, disciplina monetária, garantia dos direitos de propriedade) e as penalidades para a prática de más políticas econômicas, a liberalização da conta de capitais poderia induzir os países a seguirem políticas macroeconômicas mais disciplinadas e, desse modo, reduzir a freqüência de erros na condução da política econômica. Na medida em que a prática de políticas econômicas disciplinadas se traduza em grande estabilidade macroeconômica e financeira, o resultado pode ser altas taxas de crescimento econômico devido a uma alocação mais eficiente de recursos (Agénor, 2003. Prasad et al., 2003).

Implícitos aos argumentos supra-citados, como notado por Eatwell (1996), está a hipótese de mercados eficientes (o mercado financeiro internacional aloca capitais de forma eficiente) combinada com a primeira parte do teorema fundamental da economia do bem-estar (mercados competitivos são eficientes e, portanto, controles de capitais são ineficientes). Sem embargo, mesmo no âmbito da literatura convencional, é possível encontrar autores que chamam a atenção para a possibilidade de que os benefícios da liberalização da conta de capitais não se concretizem por conta da existência de assimetria de informação nos contratos financeiros e distorções preexistentes no funcionamento das economias domésticas (Eichengreen et al., 1998 Bailliu, 2000; Mclean \& Shrestha, 2002; Cooper, 1998).

Sob o pressuposto de existência de assimetria de informação nos contratos financeiros, ineficiências podem surgir como resultado de seleção adversa, risco moral e comportamento de manada, enfraquecendo os pressupostos teóricos a favor da liberalização da conta de capitais. A esse respeito, Eichengreen et al. (1998: 12) observam que "the classic case for unfettered capital markets is predicated on the assumption that they deliver an efficient allocation of resources. [...] under the alternative assumption that information is asymmetric, inefficiencies can arise". Mesmo quando a informação é completa, a liberalização da conta de capitais pode não resultar em estímulo ao crescimento econômico dos países, como resultado de distorções preexistentes no funcionamento das economias domésticas, notadamente, baixo nível de desenvolvimento do sistema financeiro doméstico e 
distorções em relação ao comércio internacional. ${ }^{2}$ Tendo isso em vista, Eichengreen et al. (14) afirmam que "[...] the theoretical presumption in favor of the liberalization of domestic and international financial markets is weakened by the presence of asymmetric information and domestic distortions".

No entanto, os autores afirmam que esses problemas podem ser corrigidos ou diminuídos significativamente através de políticas públicas, notadamente: i) abertura ao comércio internacional; ii) políticas que encorajem a aderência aos padrões mundiais de contabilidade e auditoria; iii) que facilitem o cumprimento de regras sadias de governança corporativa; e iv) que protejam investidores e emprestadores contra fraudes. Resulta daí que "With these important safeguards, capital account liberalization and broader financial liberalization are not only inevitable, but will clearly be beneficial" (Eichengreen et al., 1998: 1).

Ainda no âmbito da literatura convencional, uma análise bem menos otimista é apresentada por Stiglitz $(2000 ; 2003)$. O autor, tendo como pressuposto a existência de assimetria de informação nos contratos financeiros, ressalta as fragilidades teóricas subjacentes à hipótese de que a Liberalização da Conta de Capitais permitiria uma alocação mais eficiente dos recursos globais. Em adição, defende que "[...] there are even reasons to expect that capital market liberalization can have negative effects on growth" (Stiglitz, 2000: 1080).

Fora das fronteiras da ortodoxia, a literatura pós-keynesiana permite realizar uma abordagem crítica acerca dos argumentos que sublinham um conjunto de canais através dos quais a liberalização da conta de capitais estimularia o crescimento econômico dos países. Nesta perspectiva, parte-se do pressuposto de existência de incerteza fundamental, ou, usando a linguagem de Davidson (1998a), de que o sistema econômico é não ergódico, onde o futuro é incerto no sentido de que uma expectativa de ganho baseada em cálculos matemáticos confiáveis, realizados de acordo com funções de probabilidade, não pode ser obtida de algum conjunto de dados existente.

Especificamente com relação a mercados financeiros, como ressaltam Cardim de Carvalho e Sicsú (2004: 173), esse ambiente de incerteza é ainda mais importante, já que, na negociação de ativos, negociam-se, na verdade, recompensas futuras, a serem usufruídas sob condições que também só se revelarão no futuro. Não é razoável esperar que um mercado financeiro caracterizado pela existência

\footnotetext{
${ }^{2}$ Bailliu (2000: 3) argumenta que "The existing level of development of the financial system, reflected in its ability to exercise functions such as mobilizing savings, helping to allocate capital, and facilitating risk management, could play a role in determining the extent to which capital flows affect growth. The efficiency with which the domestic bank channels the borrowed funds into a productive investment project, in addition to its ability to properly evaluate the investment project, will likely influence the extent to which, if at all, this capital inflow ultimately contributes to higher economic growth". Em adição, Edison et al. (2002b: 1) argumentam que "there are innumerable constellations of distortions for which liberalization of international capital controls will hurt recourse allocation and growth. For example, in the presence of trade distortions, capital account liberalization may induce capital inflows to sectors in which the country has a comparative disadvantage".
} 
de incerteza fundamental seja eficiente para revelar os valores fundamentais subjacentes aos ativos financeiros; pelo contrário, nessa perspectiva, a função do mercado financeiro não é alocar capital de forma eficiente, mas fornecer liquidez aos agentes econômicos. Se a função do mercado financeiro não é alocar capital de forma eficiente, então não existe razão para esperar que a liberalização da conta de capitais levará a maior crescimento econômico, nem no curto nem no longo prazo. ${ }^{3}$ Portanto, "Instead of producing the utopian promises of greater stability and more rapid economic growth promised by classical economists, liberalization of capital flow regulations has been associated with exchange rate instability, slower global economic growth, and higher global unemployment" (davidson, 2003: 7).

Essa pequena síntese sugere uma interpretação alternativa à combinação ortodoxa de Hipótese de Mercados Eficientes e Economia do Bem-Estar. No entanto, nessa perspectiva teórica alternativa, não obstante o esforço de pesquisa empreendido por vários autores, as análises não apresentam uma abordagem sistemática acerca das possíveis conexões entre Liberalização da Conta de Capitais e Crescimento Econômico.

\section{A literatura empírica}

Desde o final da década de 1990 é crescente o esforço de pesquisa com o objetivo de verificar empiricamente a existência de benefícios advindos da liberalização da conta de capitais. No geral, os trabalhos estimam equações de crescimento incluindo entre as variáveis explanatórias algum indicador como proxy para o grau de liberalização da conta de capitais dos países, que varia desde proxies para a existência de restrições governamentais até a utilização dos próprios fluxos de capitais como proxy.

O trabalho pioneiro na literatura empírica foi elaborado por Quinn (1997), utilizando dados para uma amostra de países desenvolvidos e em desenvolvimento

\footnotetext{
${ }^{3}$ Davidson (1998b: 2) argumenta especificamente a esse respeito: “Since the 1970s, this 'compelling' efficient market logic has provided the justification for nations to dismantle most of the post-war capital regulation in financial markets. The argument for this 'liberalization' of financial markets was that it would produce lower real costs of capital and higher output and productivity growth rates compared to the growth rates experienced between World War II and 1973 when international capital flow controls were practiced by most countries of the world [...] In Keynes's General Theory the primary function of financial markets is to provided liquidity not efficiency, consequently real world international capital markets can never deliver, in either the short-run or the long-run, the results claimed by EMT (Efficient Market Theory)". Argumentação desenvolvida em Eatwell (1996: 35) e Alves et alli (2000), com base em fundamentos teóricos de inspiração pós-keynesiana, também sugere efeitos adversos da liberalização da conta de capitais sobre a performance econômica dos países. Bresser-Pereira e Gala (2007: 5) elaboram uma crítica ao argumento de que a liberalização da conta de capitais permitiria um aumento da eficiência na alocação internacional de recursos e resultaria em benefícios para os países em desenvolvimento: "O objetivo deste artigo é formalizar esse argumento; é fazer a crítica da política de crescimento com poupança externa hoje dominante na teoria econômica — ou, mais amplamente, do pressuposto de que os países ricos em capital devem transferir seus recursos para os países pobres em capital”.
} 
no período 1960-1989. Segundo o autor, esse estudo proporcionava a primeira demonstração sistemática de que a liberalização da conta de capitais contribui para o crescimento econômico dos países. Edison et al. (2002a), utilizando dados para uma amostra de países desenvolvidos e em desenvolvimento no período 19761995, chegam à conclusão de que os efeitos positivos da liberalização da conta de capitais são maiores e mais significativos entre os países em desenvolvimento (principalmente os países do Leste da Ásia) do que entre os países desenvolvidos. Trabalho elaborado por McLean e Shrestha (18), para uma amostra de países desenvolvidos e em desenvolvimento no período 1976-1995, cujo objetivo é testar o impacto dos diferentes fluxos de capitais sobre o crescimento econômico, chega à seguinte conclusão: "We find that foreign direct investment and portfolio inflows enhance growth".

Entre os trabalhos que não encontram evidências empíricas de efeitos positivos da liberalização da conta de capitais sobre o crescimento econômico, o primeiro foi elaborado por Rodrik (1998: 61), utilizando dados para uma amostra de países desenvolvidos e em desenvolvimento no período 1975-1989, cuja conclusão é de que "the data provide no evidence that countries without capital controls have grown faster, invested more, or experienced lower inflation", mesmo para países com alto grau de desenvolvimento institucional. Edison et al. (2000b: 24), em trabalho realizado no âmbito do FMI e utilizando dados para uma amostra de países desenvolvidos e em desenvolvimento no período 1980-1995, concluem que " The data do not support the view that international financial integration per se accelerates economic growth even when allowing this relationship to vary with economic, financial, institutional, and macroeconomic characteristics". Carkovic e Levine (2002), utilizando dados para uma amostra de países desenvolvidos e em desenvolvimento no período 1965-1995, concluem que investimento externo direto e investimento de portfolio não exercem efeito positivo sobre o crescimento econômico e que não existe uma relação causal positiva e robusta nem mesmo para países com alto grau de desenvolvimento econômico, alto grau de desenvolvimento do sistema financeiro doméstico, grande estoque de capital humano e alto grau de abertura ao comércio internacional.

Esta pequena síntese da literatura empírica sugere, no mínimo, que não existe consenso de que a liberalização da conta de capitais tenha efeitos positivos sobre o crescimento econômico dos países. ${ }^{4}$ Mais importante, os trabalhos de Edison et al. (2000b) e Carkovic e Levine (2000b) não encontram evidências de que a liberalização da conta de capitais, investimento externo direto e investimento de portfolio tenham efeitos positivos sobre o crescimento econômico, mesmo para países com alto grau de desenvolvimento econômico, alto grau de desenvolvimento institucional, alto grau de desenvolvimento do sistema financeiro doméstico, alto grau de abertura ao comércio internacional e que praticam "políticas macroeconômicas consideradas sadias".

\footnotetext{
${ }^{4}$ Uma resenha mais completa da literatura empírica pode ser encontrada em Edison et al. (2000a) e Prasad (2003).
} 


\section{CONSIDERAÇÕES METODOLÓGICAS}

\section{Especificação do modelo}

Neste trabalho será utilizado um modelo dinâmico de dados em painel. Essa abordagem parte do pressuposto de que a trajetória da taxa de crescimento é consistente com o seguinte processo (Bailliu, 2000):

$$
\begin{aligned}
& y_{i, t}=y_{i, t-1}=\lambda y_{i, t-1}+X_{i, t} \beta+u_{i, t} \\
& u_{i, t}=\mu_{i}+\eta_{t}+v_{i, t}
\end{aligned}
$$

Onde:

$y_{i, t}$ é o logaritmo do PIB real per capita do país $i$ no tempo $t, X_{i, t}$ é um vetorlinha de possíveis determinantes do crescimento econômico, $u_{i}$ é um termo não observável específico a cada país, $\eta_{t}$ é um termo não observável específico a cada período e $v_{i, t}$ é o termo aleatório. As variáveis explanatórias incluídas em $X_{i, t}$, selecionadas de acordo com a literatura empírica, são:

Investimento - Formação Bruta de Capital Fixo como proporção do PIB (Fonte: World Development Indicators 2003 - World Bank);

Governo - Consumo do governo como proporção do PIB, proxy para o tipo de política macroeconômica (Fonte: World Development Indicators 2003 World Bank);

Comércio - Importações + exportações como proporção do PIB, proxy para o grau de abertura das economias ao comércio internacional (Fonte: World Development Indicators 2003 - World Bank);

Crédito - Crédito concedido por instituições bancárias ao setor privado como proporção do PIB, proxy para o grau de desenvolvimento do sistema financeiro doméstico (Fonte: International Financial Statistics - IMF);

Inflação - Índice de preços ao consumidor, proxy para o grau de estabilidade macroeconômica (Fonte: World Development Indicators 2003 - World Bank).

Em adição, serão incluídos entre as variáveis explanatórias dois indicadores, proxies para o grau de liberalização da conta de capitais dos países:

i) GPCF - Essa variável é a soma de entrada mais saídas de fluxos de capitais privados, que incluem Investimento Externo Direto, Investimento de Portfolio e Outros Investimentos (como proporção do PIB). Essa medida de liberalização da conta de capitais é também utilizada em Edison et al. (2000b) e Prasad et al. (2003). (Fonte: World Development Indicators 2003 - World Bank);

ii) CaOpen - Essa variável foi construída por Chinn e Ito (2002), tendo como base as informações contidas no Annual Report on Exchange Arrangements and Exchange Restrictions divulgado pelo FMI. A idéia dos autores é de que este índice capte não só a existência de controles sobre a conta de capitais, mas também a intensidade desses controles, de modo que, quanto maior o valor do índice para um dado país, mais liberalizada é a conta de capitais desse país. 
A amostra utilizada no estudo é formada por 16 países da América Latina e dados anuais que cobrem o período 1986-2000. Os países presentes na amostra são: Argentina, Bolívia, Brasil, Chile, Costa Rica, El Salvador, Equador, Guatemala, Honduras, México, Panamá, Paraguai, Peru, República Dominicana, Uruguai e Venezuela.

O sinal esperado e o nível de significância dos coeficientes associados aos indicadores utilizados como proxies para o grau de liberalização da conta de capitais dos países é a hipótese a ser testada neste trabalho, ou seja, os argumentos da teoria convencional segundo os quais a liberalização da conta de capitais estimularia o crescimento econômico dos países.

\section{Abordagem econométrica}

A motivação para a utilização de um modelo dinâmico de dados em painel é a possibilidade de levar em consideração os seguintes aspectos (ver a esse respeito Blundel et al., 2000; Bond et al., 2001): i) a dimensão temporal dos dados; ii) efeitos não observáveis específicos a cada país e efeitos não observáveis específicos a cada período; iii) inclusão da variável dependente defasada entre as variáveis explanatórias; e iv) a possível endogeneidade das variáveis explanatórias. O que caracteriza a relação dinâmica é a presença da variável dependente defasada entre os regressores, o que fica claro reescrevendo a equação (1) da seguinte forma:

$$
y_{i, t}=\gamma y_{i, t-1}+X_{i, t} \beta+u_{i, t} i=1, \ldots, N \text { e } t=1, \ldots, T
$$

onde $\gamma=1+\lambda$ e, lembrando a definição de $u_{i, t}$ na equação (2), $u_{i} \sim \operatorname{IID}\left(0, \sigma_{\mu}^{2}\right)$ e $v_{i, t} \sim I I D\left(0, \sigma_{\mu}^{2}\right)$ independentes um do outro e entre eles mesmos (a especificação do modelo inclui um termo variante ao longo do tempo e constante entre os países, $\eta_{t}$, omitido nas equações apresentadas nesta seção com a finalidade de simplificar a apresentação da abordagem econométrica). Arellano e Bond (1991) sugerem um procedimento de variáveis instrumentais utilizando as condições de ortogonalidade que existem entre os valores defasados de $y_{i, t}$ e os $v_{i, t}$. O primeiro passo é fazer a primeira diferença da equação (3) para eliminar os efeitos específicos a cada país:

$$
\left(y_{i, t}-y_{i, t-1}\right)=\gamma\left(y_{i, t-1}-y_{i, t-2}\right)+\left(X_{i, t}-X_{i, t-1}\right) \beta+\left(v_{i, t}-v_{i, t-1}\right)
$$

Para o primeiro período, ou seja, $t=3$, temos:

$$
y_{i 3}-y_{i 2}=\gamma\left(y_{i 2}-y_{i 1}\right)+\left(X_{i 3}-X_{i 2}\right)+\left(v_{i 3}-v_{i 2}\right)
$$

Neste caso, $\left[y_{i 1}, X_{i 1}, X_{i 2}\right]$ são instrumentos válidos, desde que sejam altamente correlacionados com $\left(y_{i 2}-y_{i-1}\right)$ e $\left(X_{i 3}-X_{i 2}\right)$ e não correlacionados com $\left(v_{i 3}-y_{i 2}\right)$, contanto que os $v_{i, t}$ não sejam serialmente correlacionados. Para o segundo período, ou seja, $t=4$, temos:

$$
y_{i 4}-y_{i 3}=\gamma\left(y_{i 3}-y_{i 2}\right)+\left(X_{i 4}-X_{i 3}\right)+\left(v_{i 4}-v_{i 3}\right)
$$

Neste caso, $\left[y_{i 1}, y_{i 2}, X_{i 1}, X y_{i 2}, X y_{i 3}\right]$ são instrumentos válidos, desde que sejam altamente correlacionados com $\left(y_{i 3}-y_{i 2}\right)$ e $\left(X_{i 4}-X_{i 3}\right)$ e não correlacionados com $\left(v_{i 4}-v_{i 3}\right)$, 
contanto que os $v_{i, t}$ não sejam correlacionados. Continuando dessa maneira, adicionando um instrumento válido a cada período para a frente, tal que para o período $T$, o conjunto de instrumentos válidos torna-se $\left[y_{i 1}, \ldots, y_{i, T-2}, X_{i 1}, \ldots, X_{i, T-1}\right]$, obtemos a seguinte matriz de instrumentos para o país $i$ :

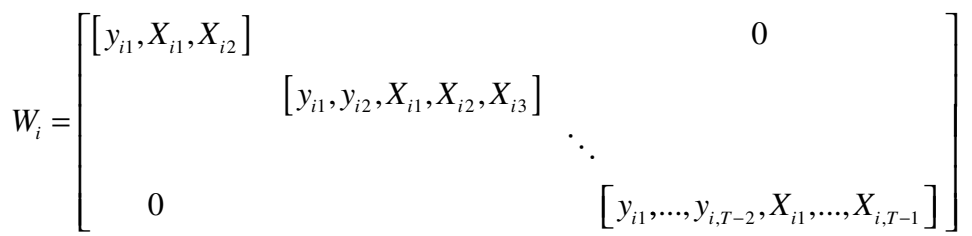

A matriz de instrumentos é $\mathrm{W}=\left[W_{1}^{\prime}, \ldots, W_{N}{ }_{N}\right]$ e as condições de momento descritas acima são dadas por $E\left(W_{i}^{\prime} \Delta v_{i}\right)=0$. Como argumenta Bond (2002: 8), o estimador GMM (Generalised Method of Moments) assintoticamente eficiente, baseado no conjunto de condições de momento estabelecido acima, minimiza o seguinte critério:

$$
J_{N}=\left[\frac{1}{N} \sum_{i=1}^{N} \Delta v_{i}^{\prime} W_{i}\right] V_{N}\left[\frac{1}{N} \sum_{i=1}^{N} W_{i}^{\prime} \Delta v_{i}\right]
$$

Onde $V_{N}$ é uma matriz de ponderação da seguinte forma:

$$
V_{N}=\left[\frac{1}{N} \sum_{i=1}^{N} W_{i}^{\prime}\left(\Delta \hat{v}_{i}\right)\left(\Delta \hat{v}_{i}\right)^{\prime} W_{i}\right]
$$

O estimador resultante é o "two-step Arellano-Bond (1991) GMM estimator”, onde os $\Delta \hat{v}_{i}$ são consistentes estimativas da primeira diferença dos resíduos obtidos de um estimador consistente preliminar. Como ressalta Baltagi (1995: 128), esse estimador GMM não requer nenhum conhecimento concernente às condições iniciais ou distribuição de $v_{i, t}$ e $\mu_{i}$.

Arellano e Bond (1991) propõem uma estatística para testar a hipótese nula de que não existe correlação serial de segunda ordem na equação em diferença. Como argumenta Baltagi (1995: 131), esse teste é importante porque a consistência do estimador GMM depende do fato de que $E\left[\Delta v_{i t} \Delta v_{i, t-2}\right]=0$. Em adição, Arellano e Bond (1991) sugerem uma estatística para testar as condições de momento utilizadas para calcular o estimador GMM, o "Sargan’s test”, sob a hipótese nula de que essas condições são válidas.

\section{APRESENTAÇÃO DOS RESULTADOS ECONOMÉTRICOS}

Antes de comentar os resultados referentes às variáveis que testam a relação entre Liberalização da Conta de Capitais e Crescimento Econômico, cabe chamar a atenção para algumas regularidades encontradas referentes às demais variáveis de controle: i) o coeficiente associado à variável Ln GDP, que é a taxa de cresci- 
mento do PIB real per capita defasada em um período, tem sinal positivo e o efeito dessa variável sobre a taxa de crescimento do PIB real per capita é estatisticamente significativo a $1 \%$ para todas as equações estimadas (Tabelas 1 e 2, em Anexos); ii) o coeficiente associado à variável Inflação tem sinal negativo e o efeito dessa variável sobre o crescimento do PIB real per capita é estatisticamente significativo a $1 \%$ para todas as equações estimadas (Tabelas 1 e 2, em Anexos); iii) o coeficiente associado à variável Investimento, que é a Formação Bruta de Capital Fixo como proporção do PIB, tem sinal positivo e o efeito dessa variável sobre o crescimento do PIB real per capita é estatisticamente significativo a $1 \%$ para todas as equações estimadas (Tabelas 1 e 2, em Anexos).

Em adição, como pode ser observado nas Tabelas 1 e 2 em Anexo, para todas as equações estimadas, não rejeitamos a hipótese nula de que as condições de momento utilizadas no estimador GMM são válidas (Sargan's test), assim como não rejeitamos a hipótese nula de que não existe correlação serial de segunda ordem (Arellano-Bond test). Ou seja, a especificação do modelo não foi rejeitada em nenhuma equação estimada.

\section{Liberalização da conta de capitais e crescimento econômico}

$\mathrm{O}$ sinal esperado para os coeficientes associados às variáveis $G P C F$ e $\mathrm{Ca}$ OPEN, nas colunas A1 e A2, respectivamente, de acordo com a teoria convencional, é positivo, o que significaria que a liberalização da conta de capitais estimula o crescimento econômico. No entanto, como pode ser observado na coluna A1 da Tabela 1, o coeficiente associado a GPCF tem sinal negativo e o efeito dessa variável sobre o crescimento do PIB real per capita é estatisticamente significativo a $1 \%$. Como observado na coluna A2 da Tabela 1 , o coeficiente associado a $\mathrm{Ca}$ OPEN tem sinal negativo e o efeito dessa variável sobre o crescimento do PIB real per capita não é estatisticamente significativo. Portanto, de acordo com os resultados encontrados para essa amostra de países, período e metodologia econométrica utilizada, não se pode aceitar a hipótese de que a liberalização da conta de capitais estimula o crescimento econômico, sugerindo, ao contrário, um efeito adverso sobre a taxa de crescimento do PIB real per capita.

Como os resultados não mostram uma relação causal positiva e robusta da liberalização da conta de capitais para o crescimento do PIB real per capita, a próxima etapa do trabalho é testar um conjunto de hipóteses anunciadas na literatura convencional, segundo as quais o efeito da liberalização da conta de capitais dependeria do grau de abertura das economias ao comércio internacional, grau de desenvolvimento do sistema financeiro doméstico, tipo de política macroeconômica praticada e grau de estabilidade macroeconômica das economias. Essas hipóteses serão testadas nas próximas subseções através da construção de variáveis de interação entre os indicadores de liberalização da conta de capitais (GPCF e $\mathrm{CaOpen)}$ e o indicador de abertura ao comércio internacional (Comércio), desenvolvimento do sistema financeiro doméstico (Crédito), políticas macroeconômicas consideradas sadias (Governo) e estabilidade macroeconômica (Inflação). 


\section{Liberalização da conta de capitais, abertura comercial e crescimento econômico}

Como exposto na seção 2, alguns autores argumentam que, na presença de distorções com relação ao comércio internacional, a liberalização da conta de capitais pode ter efeitos adversos sobre o crescimento econômico, isso porque na presença de barreiras ao comércio os influxos de capitais podem exacerbar a má alocação de capital entre setores intensivos em capital e setores intensivos em trabalho, levando a uma alocação ineficiente dos recursos externos.

Essa hipótese é testada, como em Carkovic e Levine (2002), construindo variáveis de interação entre os indicadores de liberalização da conta de capitais e o indicador de abertura comercial (GPCF $x$ Comércio e CaOPEN $x$ Comércio), o que equivale a testar se à medida que a economia se torna mais aberta ao comércio internacional o efeito da liberalização da conta de capitais sobre o crescimento econômico é potencializado e, portanto, de acordo com a literatura convencional, espera-se que o sinal dos coeficientes associados a essas variáveis de interação sejam positivos e estatisticamente significativos. Os resultados são apresentados nas colunas B1 e B2 da Tabela 1.

Como pode ser observado na coluna B1 da Tabela 1, o coeficiente associado à variável interativa GPCF $x$ Comércio tem sinal positivo, mas o efeito dessa variável sobre o crescimento do PIB real per capita não é estatisticamente significativo a $10 \%$. Quanto à variável CaOPEN $x$ Comércio, coluna B2 da Tabela 1 , o sinal do coeficiente associado a ela é positivo e o efeito dessa variável sobre o crescimento do PIB real per capita é estatisticamente significativo a 5\%. Desse modo, os resultados encontrados nos cálculos econométricos não são conclusivos, não permitindo rejeitar fortemente a hipótese de que o efeito da liberalização da conta de capitais sobre o crescimento econômico depende do grau de abertura das economias ao comércio internacional.

\section{Liberalização da conta de capitais, sistema financeiro e crescimento econômico}

Como exposto na seção 2, alguns autores argumentam que a liberalização da conta de capitais na presença de um setor financeiro desenvolvido pode ter seus efeitos sobre o crescimento econômico potencializados, isso porque um sistema financeiro desenvolvido seria mais eficiente em converter recursos externos em investimentos produtivos e mais hábil para alocá-los nos projetos de investimento mais produtivos. Caso contrário, a liberalização da conta de capitais na presença de um sistema financeiro doméstico pouco desenvolvido pode exacerbar problemas decorrentes da existência de assimetrias de informação, levando a uma alocação ineficiente dos recursos externos e, desse modo, podendo ter efeito adverso sobre o crescimento econômico (Bailliu).

Para testar essa hipótese - como em Carkovic e Levine (2002) e Edison et al. (2002b) - foram construídas variáveis de interação entre os indicadores de liberalização da conta de capitais e o indicador de desenvolvimento do sistema finan- 
ceiro doméstico (GPCF $x$ Crédito e CaOPEN $x$ Crédito), o que equivale a testar se à medida que aumenta o grau de desenvolvimento do sistema financeiro doméstico o efeito da liberalização da conta de capitais sobre o crescimento econômico é potencializado e, portanto, de acordo com a literatura convencional, o sinal esperado para o coeficiente associado a essas variáveis de interação é positivo.

Como pode ser observado nas colunas A1 e A2 da Tabela 2, os coeficientes das variáveis de interação GPCF $x$ Crédito e CaOPEN $x$ Crédito apresentam sinais negativos e o efeito dessas variáveis sobre o crescimento do PIB real per capita é estatisticamente significativo a $5 \%$ e $1 \%$, respectivamente, em desacordo com o que seria esperado pela literatura convencional. Portanto, os resultados dos cálculos econométricos não corroboram a hipótese de que o efeito da liberalização da conta de capitais sobre o crescimento econômico seja potencializado pelo desenvolvimento do sistema financeiro doméstico dos países.

\section{Liberalização da conta de capitais, política macroeconômica e crescimento econômico}

Eichengreen et al. (1998) sugerem como pré-requisito para potencializar os efeitos positivos da liberalização da conta de capitais sobre o crescimento econômico a prática de políticas macroeconômicas consideradas "sadias". Para testar essa hipótese - como em Edison et al. (2002b) - são construídas variáveis de interação entre os indicadores de liberalização da conta de capitais e a proxy para o tamanho do governo na economia (GPCF $x$ Governo e CaOPEN $x$ Governo). A idéia é que o aumento da participação do governo na economia, ao gerar distorções, tende a diminuir os possíveis efeitos positivos da liberalização da conta de capitais sobre o crescimento econômico e, portanto, o sinal esperado para os coeficientes associados a essas variáveis de interação é negativo.

Como pode ser observado na coluna B1 da Tabela 2, o coeficiente associado à variável interativa GPCF $x$ Governo apresenta sinal positivo (o que é contraditório ao esperado pela literatura convencional) e o efeito dessa variável sobre o crescimento do PIB real per capita não é estatisticamente significativo. O coeficiente associado à variável CaOPEN $x$ Governo, como pode ser observado na coluna B2 da Tabela 2, apresenta sinal negativo e o efeito dessa variável sobre o crescimento do PIB real per capita não é estatisticamente significativo. Desse modo, os resultados encontrados não corroboram a hipótese de que o efeito da liberalização da conta de capitais sobre o crescimento econômico dependa da prática de políticas macroeconômicas consideradas "sadias" pela literatura convencional.

\section{Liberalização da conta de capitais, estabilidade macroeconômica e crescimento econômico}

Por fim, a última hipótese a ser testada é se o efeito da liberalização da conta de capitais sobre o crescimento econômico depende da estabilidade macroeconômica. Como em Edison et al. (2000b), essa hipótese é testada construindo variáveis 
de interação entre os indicadores de liberalização da conta de capitais e a variável Inflação, proxy para estabilidade macroeconômica (GPCF $x$ Inflação e CaOPEN $x$ Inflação). A idéia é que o aumento da instabilidade macroeconômica tende a diminuir os potenciais benefícios da liberalização da conta de capitais e, portanto, o sinal esperado para o coeficiente associado a essas variáveis é negativo.

Como pode ser observado nas colunas C1 e C2 da Tabela 2, os coeficientes associados às variáveis de iteração GPCF $x$ Inflação e CaOPEN $x$ Inflação têm sinal negativo, o que está de acordo ao esperado pela literatura convencional. No entanto, o efeito dessas variáveis sobre o crescimento do PIB real per capita não é estatisticamente significativo a $10 \%$. Portanto, os resultados dos cálculos econométricos não corroboram a hipótese de que o efeito da liberalização da conta de capitais sobre o crescimento econômico dependa da estabilidade macroeconômica.

\section{CONSIDERAÇÕES FINAIS}

Os cálculos econométricos apresentados na seção 4, em consonância com a falta de consenso verificada na literatura empírica, não corroboram a hipótese de que a liberalização da conta de capitais estimula o crescimento econômico. Os resultados para essa amostra de países, período, metodologia econométrica e indicadores utilizados como proxies para o grau de liberalização da conta de capitais dos países sugerem um efeito adverso da liberalização da conta de capitais sobre o crescimento do PIB real per capita. Em adição, foram testadas hipóteses para averiguar se a relação entre Liberalização da Conta de Capitais e Crescimento Econômico depende do grau de abertura das economias ao comércio internacional, desenvolvimento do sistema financeiro doméstico, prática de políticas econômicas consideradas "sadias" pela literatura convencional e estabilidade macroeconômica. Os cálculos econométricos mostraram os seguintes resultados: i) os resultados não são conclusivos, não permitindo rejeitar fortemente a hipótese de que o efeito da liberalização da conta de capitais sobre o crescimento econômico depende do grau de abertura das economias ao comércio internacional; ii) os resultados não corroboram a hipótese de que a relação entre Liberalização da Conta de Capitais e Crescimento Econômico depende do grau de desenvolvimento do sistema financeiro doméstico, prática de políticas econômicas consideradas "sadias" pela literatura convencional e estabilidade macroeconômica.

Em estudo supracitado, elaborado no âmbito do FMI, Prasad et al. (2003: p. 37) questionam "why is it so difficult to find a strong and robust effect of financial integration on economic growth for developing countries, when the theoretical basis for this result is apparently so strong?" A resposta de Prasad et al. (2003: p. 37) é sumarizada na seguinte frase: "Of course, the lack of a strong and robust effect of financial integration on economic growth does not necessarily imply that theories that make this connection are wrong. Financial Integration without a proper set of preconditions might lead to few growth benefits [...]”. Essa é a hipótese que 
emerge da revisão da literatura convencional realizada na seção 2; ao considerar as implicações da existência de assimetria de informação no mercado financeiro e distorções no funcionamento das economias domésticas para a relação entre liberalização da conta de capitais e crescimento econômico, seria necessário um conjunto de precondições a ser preenchido pelas economias nacionais para que os potenciais benefícios da liberalização da conta de capitais se concretizassem: i) certo nível de desenvolvimento do sistema financeiro doméstico; ii) certo grau de abertura ao comércio internacional; iii) prática de políticas econômicas consideradas "sadias" pela literatura convencional; e iv) estabilidade macroeconômica.

Nesse sentido, os resultados econométricos apresentados neste trabalho para os países da América Latina, em consonância com os resultados encontrados nos trabalhos de Edison et al. (2002b) e Carkovic e Levine (op. cit.,), não dão sustentação empírica para a hipótese de benefícios advindos da liberalização da conta de capitais, bem como também não corroboram a hipótese de que seria necessário um conjunto de precondições a ser preenchido pelas economias domésticas para que os benefícios da liberalização da conta de capitais se concretizem. Ou seja, os resultados econométricos apresentados neste trabalho e nos dois trabalhos, citados acima, representam um questionamento da idéia de seqüência ótima das reformas econômicas. Isso justifica a apreciação crítica da literatura teórica feita na seção 2, ao deixar subjacente o questionamento de se são realmente robustas as teorias que estabelecem uma relação causal positiva da liberalização da conta de capitais para o crescimento econômico.

\section{REFERÊNCIAS BIBLIOGRÁFICAS}

AGÉNOR, Pierre-Richard. Benefits and Costs of International Financial Integration: Theory and Facts. Washington, The World Bank, 2003.

ALVES Jr., A. J.; FERRARI FILHO, F.; PAULA, L. F. The Post Keynesian critique of conventional currency crisis models and Davidson's proposal to reform the international monetary System. Journal of Post Keynesian Economics, 22 (2), p.209-227, Inverno, 1999-2000.

ARELLANO, M.; BOND, S. Some Tests of Specification for Panel Data: Monte Carlo Evidence and an Application to Employment Equations. Review of Economic Studies, 58, 277-297, 1991.

BAILLIU, J. N. Private Capital Flows, Financial Development, and Economic Growth in Developing Countries. Ottawa, Bank of Canada, Working Paper, 2000.

BALTAGI, B. H. Econometric Analysis of Panel Data. England: John Wiley \& Sons, 1995.

BLUNDELL, R.; BOND, S.; WINDMEIJER, F. Estimation in Dynamic Panel Data Models: Improving on the Performance of The Standard GMM Estimators. The Institute for Fiscal Studies, Working Paper 12, 2000.

BOND, S.; HOEFFLER, A.; TEMPLE, J. GMM Estimation of Empirical Growth Models. University of Oxford, Institute for Fiscal Studies, 2001.

BRESSER-PEREIRA, L. C.; GALA, P. Por Que a Poupança Externa não Promove Crescimento. Revista de Economia Política, v.27, n.1 (105), p.3-19, janeiro-março/2007.

CARKOVIC, M.; LEVINE, R. Does Foreign Direct Investment Accelerate Economic Growth? University of Minnesota, 2002. 
CARDIM DE CARVALHO, F.; SICSÚ, J. Controvérsias Recentes Sobre Controles de Capitais. Revista de Economia Política, v.24, n.2 (94), p.163-184, abril-junho/2004.

CARDIM DE CARVALHO, F. Controles de Capitais: Uma Agenda de Pesquisa. Revista de Economia Contemporânea, Rio de Janeiro, 8 (2), p. 283-298, julho-dezembro/2004.

CARDIM DE CARVALHO, F.; SICSÚ, J. Teorias e Experiências de Controles do Fluxo de Capitais: Focando o Caso da Malásia. Economia e Sociedade, Campinas, v.14, n.2 (25), p.365-374, julhodezembro/2005.

CHINN, M.; ITO, H. Capital Account Liberalization, Institutions and Financial Development: Cross Country Evidence. NBER Working Paper \#8967, 2002.

COOPER, R. N. Should Capital-Account Convertibility Be a World Objective? In: PETER, B. Kenen (ed). Should the IMF Pursue Capital-Account Convertibility. Princeton University, Department of Economics, Essays in international Finance $n^{\circ} 207,1998$.

DAVIDSON, P. Volatile Financial Markets and The Speculator. Paper Presented as The Economic Issues Lecture to the Royal Economic Society Annual Conference, Warwick, England, April 1998a.

DAVIDSON, P. The Case For Regulating International Capital Flows. Paper Presented at the Social Market Foundation Seminar on Regulation of Capital Movements, London, November 17, 1998b.

DAVIDSON, P. Financial Markets, Money and the Real World. Massachusetts: Edward Elgar, 2002.

DAVIDSON, P. The Future of The International Financial System. Paper Presented at Conference on the Future of Economics at Cambridge University, Selptember 18, 2003.

EATWELL, John. International Financial Liberalization: The Impact on World Development. New York: CEPA Working Paper ${ }^{\circ}$ 1, 1996.

EDISON, H. J.; KLEIN, M.W.; RICCI, L.; SLOEK, T. Capital Account Liberalization and Economic Performance: Survey and Synthesis. Massachusetts, National Bureau of Economic Research, Working Paper 9100, 2002a.

EDISON, H.J.; LEVINE, R.; RICCI, L.; SLOEK, T. International Financial Integration and Economic Growth. Massachusetts, National Bureau of Economic Research, Working Paper 9164, 2002b.

EICHENGREEN, B.; MUSSA, M.; DELL'ARICCIA, G.; DETRAGIACHE, E.; MILESI-FERRETTI, G.; TWEEDIE, A. Capital Account Liberalization: Theoretical and Practical Aspects. Washington, International Monetary Fund, Occasional Paper nº 172, 1998.

INTERNATIONAL MONETARY FOUND. International Financial Statistics. CD-ROM, 2003.

KLEIN, M; OLIVEI G. Capital Account Liberalization, Financial Depth, and Economic Growth. Massachusetts, National Bureau of Economic Research, Working Paper 7384, 1999.

McLEAN, B.; SHRESTHA, S. International Financial Liberalization and Economic Growth. Reserve Bank of Australia, Research Discussion Paper n 3, 2002.

OBSTFELD, M.; TAYLOR, A. M. Global Capital Markets: Integration, Crisis, and Growth. Cambridge University Press, 2004.

OBSTFELD, M. Risk-Taking, Global Diversification and Growth. American Economic Review, 84(5), p.1310-1329, 1994.

PRASAD, E.; ROGOFF, K.; WEI, S.; KOSE, M. K. Effects of Financial Globalization on Developing Countries: Some Empirical Evidence. International Monetary Fund, 2003.

QUINN, D. The Correlates of Change in international Financial Regulation. American Political Science Review, vol. 91, n 3, p. 531-551, 1997.

REISEN, H.; SOTO, M. Which Types of Capital Inflows Foster Developing-Country Growth. International Finance 4:1, p.1-14, 2001.

RODRIK, D. Who Needs Capital-Account Convertitility? In: PETER, B. K. (ed). Should the IMF Pursue Capital-Account Convertitility. Princeton University, Department of Economics, Essays in International Finance ${ }^{\circ} 207,1998$.

ROGOFF, K. Managing the World Economy. The Economist, 03/08/2002. 
STIGLITZ, J. Capital Market Liberalization, Economic Growth, and Instability. Washington, World Bank, World Development v. 28, n. 6, p.1075-1086, 2000.

STIGLITZ, J. Globalization and Growth in Emerging Markets and the New Economy. Journal of Policy Modeling, v. 25, p.505-524, 2003.

WORLD BANK. World Development Indicators. CD-ROM, 2003.

\section{ANEXOS}

Tabela 1: Liberalização de conta de capitais e crescimento econômico (Variável dependente: crescimento do PIB real per capita)

\begin{tabular}{|c|c|c|c|c|}
\hline \multirow[b]{2}{*}{ Variáveis Explanatórias } & \multicolumn{2}{|c|}{ A } & \multicolumn{2}{|c|}{$\mathrm{B}$} \\
\hline & A1 & $\mathrm{A} 2$ & B1 & $\mathrm{B} 2$ \\
\hline Ln GDP & $\begin{array}{r}0,82966 \\
(0,032)^{*}\end{array}$ & $\begin{array}{r}0,81073 \\
(0,033)^{*}\end{array}$ & $\begin{array}{r}0,82238 \\
(0,031)^{*}\end{array}$ & $\begin{array}{r}0,79722 \\
(0,032)^{*}\end{array}$ \\
\hline Inflação & $\begin{array}{r}-0,00001 \\
(0,000)^{*}\end{array}$ & $\begin{array}{c}-0,00001 \\
(0,000)^{*}\end{array}$ & $\begin{array}{c}-0,00001 \\
(0,000)^{*}\end{array}$ & $\begin{array}{r}-0,00001 \\
(0,000)^{*}\end{array}$ \\
\hline Comércio & $\begin{array}{c}0,00606 \\
(0,028)\end{array}$ & $\begin{array}{c}0,01488 \\
(0,029)\end{array}$ & $\begin{array}{c}-0,00898 \\
(0,030)\end{array}$ & $\begin{array}{c}-0,021851 \\
(0,030)\end{array}$ \\
\hline Governo & $\begin{array}{r}0,13267 \\
(0,116)^{*}\end{array}$ & $\begin{array}{c}0,05215 \\
(0,118)\end{array}$ & $\begin{array}{c}0,12018 \\
(0,115)\end{array}$ & $\begin{array}{c}0,17074 \\
(0,134)\end{array}$ \\
\hline Investimento & $\begin{array}{c}0,26183 \\
(0,898)^{*}\end{array}$ & $\begin{array}{c}0,33138 \\
(0,091)^{*}\end{array}$ & $\begin{array}{c}0,25969 \\
(0,089)^{*}\end{array}$ & $\begin{array}{c}0,34625 \\
(0,089)^{*}\end{array}$ \\
\hline Crédito & $\begin{array}{c}-0,0156 \\
(0,022)\end{array}$ & $\begin{array}{c}-0,01129 \\
(0,022)\end{array}$ & $\begin{array}{c}-0,01481 \\
(0,021)\end{array}$ & $\begin{array}{c}-0,00744 \\
(0,022)\end{array}$ \\
\hline GPCF & $\begin{array}{c}-0,02574 \\
(0,005)^{*}\end{array}$ & & $\begin{array}{c}-0,03675 \\
(0,021)^{* * * *}\end{array}$ & \\
\hline CaOpen & & $\begin{array}{c}-0,00400 \\
(0,002)\end{array}$ & & $\begin{array}{c}-0,01549 \\
(0,006)^{*}\end{array}$ \\
\hline GPCF x Comércio & & & $\begin{array}{c}0,05151 \\
(0,093)\end{array}$ & \\
\hline CaOPEN x Comércio & & & & $\begin{array}{r}0,03013 \\
(0,013)^{* *} \\
\end{array}$ \\
\hline Sargan Test (valor-p) & 1,0000 & 1,0000 & 1,0000 & 1,0000 \\
\hline Arell.-Bond Test (valor-p) & 0,1785 & 0,1376 & 0,727 & 0,1624 \\
\hline No de Observações & 208 & 208 & 208 & 208 \\
\hline
\end{tabular}

Notas:

a: Constante não reportada presente em todas as regressões;

b: Todas as variáveis explanatórias estão em primeira diferença;

c: Desvio-padrão de todas as variáveis explanatórias em parênteses;

$\mathrm{d}:{ }^{*},{ }^{* *},{ }^{* * *}$, significativos a $1 \%, 5 \%$ e $10 \%$, respectivamente. 
Tabela 2: Liberalização de conta de capitais e crescimento econômico (Variável dependente: crescimento do PIB real per capita)

\begin{tabular}{|c|c|c|c|c|c|c|}
\hline \multirow[b]{2}{*}{ Variáveis Explanatórias } & \multicolumn{2}{|c|}{ A } & \multicolumn{2}{|c|}{ B } & \multicolumn{2}{|c|}{ C } \\
\hline & $\mathrm{A} 1$ & $\mathrm{~A} 2$ & B1 & B2 & $\mathrm{C} 1$ & $\mathrm{C} 2$ \\
\hline Ln GDP & $\begin{array}{l}0,8641 \\
(0,031)^{*}\end{array}$ & $\begin{array}{r}0,77784 \\
(0,033)^{*}\end{array}$ & $\begin{array}{c}0,08254 \\
(0,031)^{*}\end{array}$ & $\begin{array}{c}0,79866 \\
(0,033)^{*}\end{array}$ & $\begin{array}{c}0,82195 \\
(0,027)^{*}\end{array}$ & $\begin{array}{c}0,79169 \\
(0,031)^{*}\end{array}$ \\
\hline Inflação & $\begin{array}{c}-0,00001 \\
(0,000)^{*}\end{array}$ & $\begin{array}{c}-0,00001 \\
(0,000)^{*}\end{array}$ & $\begin{array}{r}-0,00001 \\
(0,000)^{*}\end{array}$ & $\begin{array}{c}-0,00001 \\
(0,000)^{*}\end{array}$ & $\begin{array}{l}-0,00001 \\
(0,000)^{* * * *}\end{array}$ & $\begin{array}{r}-0,00009 \\
(0,000)^{*}\end{array}$ \\
\hline Comércio & $\begin{array}{c}-0,00656 \\
(0,027)\end{array}$ & $\begin{array}{c}0,00759 \\
(0,028)\end{array}$ & $\begin{array}{c}-0,00066 \\
(0,028)\end{array}$ & $\begin{array}{c}0,00588 \\
(0,028)\end{array}$ & $\begin{array}{c}0,00051 \\
(0,027)\end{array}$ & $\begin{array}{c}0,00833 \\
(0,027)\end{array}$ \\
\hline Governo & $\begin{array}{c}0,07311 \\
(0,117)\end{array}$ & $\begin{array}{c}0,06197 \\
(0,117)\end{array}$ & $\begin{array}{c}0,10055 \\
(0,121)\end{array}$ & $\begin{array}{c}0,02726 \\
(0,117)\end{array}$ & $\begin{array}{c}0,09889 \\
(0,118)\end{array}$ & $\begin{array}{c}-0,11261 \\
(0,122)\end{array}$ \\
\hline Investimento & $\begin{array}{c}0,36083 \\
(0,096)^{*}\end{array}$ & $\begin{array}{c}0,47205 \\
(0,098)^{*}\end{array}$ & $\begin{array}{c}0,27229 \\
(0,089)^{*}\end{array}$ & $\begin{array}{c}0,34246 \\
(0,091)^{*}\end{array}$ & $\begin{array}{c}0,25717 \\
(0,090)^{*}\end{array}$ & $\begin{array}{c}0,287789 \\
(0,089)^{*}\end{array}$ \\
\hline Crédito & $\begin{array}{c}0,00786 \\
(0,024)\end{array}$ & $\begin{array}{c}-0,02287 \\
(0,022)\end{array}$ & $\begin{array}{c}-0,01301 \\
(0,022)\end{array}$ & $\begin{array}{c}-0,00968 \\
(0,022)\end{array}$ & $\begin{array}{c}-0,01063 \\
(0,022)\end{array}$ & $\begin{array}{c}-0,00338 \\
(0,021)\end{array}$ \\
\hline GPCF & $\begin{array}{c}0,05471 \\
(0,034)\end{array}$ & & $\begin{array}{c}-0,05156 \\
(0,050)\end{array}$ & & $\begin{array}{c}-0,02495 \\
(0,005)^{*}\end{array}$ & \\
\hline CaOpen & & $\begin{array}{c}0,00006 \\
(0,003)\end{array}$ & & $\begin{array}{c}-0,00124 \\
(0,007)\end{array}$ & & $\begin{array}{c}-0,00336 \\
(0,002)\end{array}$ \\
\hline GPCF x Crédito & $\begin{array}{r}-0,18388 \\
(0,077)^{* *}\end{array}$ & & & & & \\
\hline CaOPEN x Crédito & & $\begin{array}{c}-0,03552 \\
(0,010)^{*}\end{array}$ & & & & \\
\hline GPCF x Governo & & & $\begin{array}{c}0,12276 \\
(0,235)\end{array}$ & & & \\
\hline CaOPEN x Governo & & & & $\begin{array}{c}-0,01869 \\
(0,064)\end{array}$ & & \\
\hline GPCF x Inflação & & & & & $\begin{array}{c}-0,00007 \\
(0,001)\end{array}$ & \\
\hline CaOPEN x Inflação & & & & & & $\begin{array}{c}-0,00004 \\
(0,026)\end{array}$ \\
\hline Sargan Test (valor-p) & 1,0000 & 1,0000 & 1,0000 & 1,0000 & 1,0000 & 1,0000 \\
\hline Arell.-Bond Test (valor-p) & 0,1113 & 0,1682 & 0,2009 & 0,1410 & 0,2327 & 0,2547 \\
\hline$N^{\circ}$ de Observações & 208 & 208 & 208 & 208 & 208 & 208 \\
\hline
\end{tabular}

\section{Notas:}

a: Constante não reportada presente em todas as regressões;

b: Todas as variáveis explanatórias estão em primeira diferença;

c: Desvio-padrão de todas as variáveis explanatórias em parênteses;

$\mathrm{d}:{ }^{*}, * * * * * *$, significativos a $1 \%, 5 \%$ e $10 \%$, respectivamente. 